information and proportionately distributes ill-defined ICD codes among disease groups without explicit guidelines. We propose a modified classification system-Million Death Study mortality classification system (MDS-MC) - that is practicable for use in verbal autopsy studies worldwide.

Methods We use a Delphi method to categorise ICD codes into disease categories. These categories are then structured into broader disease groups. We compare our classification system against GBD and check for age and sex-wise epidemiological plausibility of major diseases using the MDS study results for 123000 deaths from 2001 to 2003 across India.

Results 31 disease groups are divided into four broad disease group$s$-communicable, non-communicable, injury and ill-defined. The MDS-MC produces built-in quality indicators (such as proportion of ill-defined causes for deaths before old age, range checks, etc) that make it easier to manage the inherent misclassification in verbal autopsy. Major disease profiles are epidemiologically plausible for age and sex. A simple to use web-program enables its use in various settings.

Conclusions The MDS-MC, designed specifically for coding of verbal autopsies, is a widely practicable classification system for use in developing countries.

\section{6-5.2 LINKING INDIVIDUAL RECORDS FROM MULTIPLE LARGE DATABASES; A SHORT HISTORY AND PRACTICAL SOLUTION}

doi:10.1136/jech.2011.142976b.85

M Lugg.* Kaiser Permanente, Pasadena, California, USA

Quality epidemiological studies require linked records to differentiate persons from events.

Medical records in the 1960's were mainly linked manually by visually comparing personal data, such as in the Western Australian Mental Health Services Registry.

In 1973, Australia's Hospital and Allied Services Advisory Council (HASAC) studied numerous ways of creating a uniform personal identifier using personal data / attributes which were readily available to the patient and healthcare provider without recalling a medical record number or finding a membership card. Using portions of the last name, first name, gender, and date of birth; only 17 out of 696000 records could not be automatically linked. A tiebreaker, such as place of birth was recommended.

In 1993, the California Health Information for Policy Project's Interagency Working Party, working with 10 different state and local databases, suggested the use of the HASAC algorithm, but used mother's maiden name instead of place of birth to prevent duplicates requiring manual adjudication. This recommendation however could not be implemented practically because many organisations whose records did not include mother's maiden name found the cost of finding this information prohibitive.

In 2003, an attempt was made to further modify the HASAC criteria using only computerised available data to link just under 2 million records for the Kaiser Permanente Southern California Immunisation registry (KITS). As series of 26 computerised algorithms was developed which resulted in a $99.8 \%$ matching rate.

This study resulted in a cost-effected way of routinely linking records from large data bases with minimal manual input.

\section{6-5.3 VASOVAGAL REACTION FROM BLOOD DONATION AND BIOMARKERS IN JAPANESE}

doi:10.1136/jech.2011.142976b.86

${ }^{1} \mathrm{~T}$ Odajima, ${ }^{*}{ }^{1} \mathrm{H}$ Sugimori, ${ }^{2} \mathrm{M}$ Takanashi, ${ }^{2} \mathrm{~S}$ Aota, ${ }^{2} \mathrm{M}$ Sudoh, ${ }^{2} \mathrm{Y}$ Yamaga, ${ }^{2} \mathrm{Y}$ Ono, ${ }^{2} \mathrm{~K}$ Matsuzaki, ${ }^{2,3} \mathrm{M}$ Satake, ${ }^{4} \mathrm{~K}$ Yoshinaga, ${ }^{4} \mathrm{~T}$ Motoji, ${ }^{2} \mathrm{~K}$ Nakajima. ${ }^{1}$ Department of Preventive Medicine, Daito Bunka University Graduate School of Sports and Health
Science, Saitama, Japan; ${ }^{2}$ Japanese Red Cross Tokyo Blood Centre, Tokyo, Japan: ${ }^{3}$ Japanese Red Cross Central Blood Institute, Tokyo, Japan; ${ }^{4}$ Department of Hematology, Tokyo Women's Medical University, Tokyo, Japan

Introduction The Japanese Red Cross Tokyo Blood Center collects approximately 600000 blood donations every year. Since we experienced a fatal injury case related to vasovagal reaction (VVR) syncope in 2005, we conducted a cross-sectional study to elucidate factors contributing to VVR, the most frequent side effect, following whole blood and apheresis donations.

Methods Complications recorded at the collection sites after voluntary donations by Japanese Red Cross Tokyo Blood Center, in the 2006 and 2007 fiscal years, were analysed by univariate and multivariate logistic regression model. Of 1119716 blood donations over a full 2 years, complications were recorded for 13320 donations $(1.189 \%)$, among which VVR was the primary or secondary complication in $67 \%$. Eligible 4303 VVR cases having sufficient information, and 40256 control donors were prepared. Age, body mass index, predonation blood pressure, pulse, and biomarkers (eg, total protein, albumin, and haemoglobin) were compared between VVR group and control group.

Results VVR group was significantly younger, lower body mass index, lower blood pressure, higher biomarkers' values (eg, total protein, albumin, and haemoglobin) than control group. $(p<0.001)$ Furthermore, biomarkers' values and VVR incidences showed a dosedependent manner. (Trend test $\mathrm{p}<0.01$ ).

Conclusion Obviously, to prevent serious consequences, donors should be informed about importance of rest afterwards, and posture to take when symptoms occur. From our analysis, extra care should be considered including high biomarkers' concentrations (eg, total protein, albumin, and haemoglobin), which might reflect donor's dehydration state.

\section{6-5.4 VENTILATOR-ASSOCIATED PNEUMONIA IN PATIENTS WITH SEVERE BURN INJURY: THE PREDICTIVE VALUE OF ROUTINE SURVEILLANCE CULTURES TO PREDICT MULTIDRUG RESISTANCE}

doi:10.1136/jech.2011.142976b.87

${ }^{1,2} \mathrm{~N}$ Brusselaers, ${ }^{*}{ }^{1} \mathrm{D}$ Logie, ${ }^{1,2} \mathrm{D}$ Vogelaers, ${ }^{1,3} \mathrm{~S}$ Monstrey, ${ }^{1,2} \mathrm{~S}$ Blot. ${ }^{1}$ Ghent University, Ghent, Belgium; ${ }^{2}$ Ghent University Hospital, General Internal Medicine, Ghent, Belgium; ${ }^{3}$ Ghent University Hospital, Burn Unit, Ghent, Belgium

Introduction Burn patients with inhalation injury requiring mechanical ventilation $(\mathrm{MV})$ are at particular risk for ventilatorassociated pneumonia (VAP), which is associated with increased morbidity and mortality. Routine endotracheal surveillance cultures (SC) may provide information about the causative pathogen in subsequent VAP, facilitating early appropriate antibiotic therapy.

Objectives To assess the value of routine endotracheal SC to predict multidrug resistant (MDR) aetiology of VAP in burn patients with inhalation injury.

Methods Historical cohort ( $\mathrm{N}=46)$ study including all burn patients with inhalation injury who developed VAP during admission to the burn unit at Ghent University Hospital (2002-2009).

Results Overall, 70 episodes of VAP occurred. Median age and total burned surface area were 43.5 y (IOR 38.0 to 54.3 ) and 32.5\% (18.0 to 45.8$)$ respectively. The median Belgian Outcome in Burn Injury score was 5 (4-6), reflecting a predicted mortality of $30 \%$ (20-50\%). ${ }^{1}$ Median duration of MV prior to onset VAP was $7 \mathrm{~d}$ (4-9d). The incidence of VAP was 55 episodes/1000 MV days and 112 episodes/1000 MV days "at risk." In 23 episodes (32.9\%) at least one MDR causative pathogen was involved (24 MDR pathogens), mostly Pseudomonas aeruginosa (10/23) and Enterobacter spp. (7/23). The sensitivity and specificity of SC to predict MDR pathogens was 
respectively $83.0 \%$ and $96.2 \%$, corresponding with a positive predictive value of $87.0 \%$ and a negative predictive value of $95.0 \%$. Subgroup analyses showed higher predictive values for second VAP episodes, and when $P$ aeruginosa was involved.

Conclusions In this cohort routine SC appear to have excellent operating characteristics to predict MDR involvement in VAP.

\section{REFERENCE}

1. Br J Surg 2008;96:111-17.

\section{6-5.5 ORAL MISOPROSTOL IN PREVENTING POSTPARTUM HAEMORRHAGE AT HOME BIRTH IN RURAL BANGLADESH: HOW EFFECTIVE IT IS?}

doi:10.1136/jech.2011.142976b.88

${ }^{1} \mathrm{H}$ E Nasreen, ${ }^{*}{ }^{2} \mathrm{~S}$ Nahar, ${ }^{1} \mathrm{M}$ Mamun, ${ }^{1} \mathrm{~K}$ Afsana, ${ }^{3} \mathrm{P}$ Byass. ${ }^{1} B R A C$, Dhaka, Bangladesh; ${ }^{2}$ King Khalid University, Abha, Saudi Arabia; ${ }^{3}$ Umea University, Umea, Sweden

Aims Insufficient evidence exists regarding the prevention of postpartum haemorrahge $(\mathrm{PPH})$ by oral administration of misoprotol in low income countries. This study investigates whether $400 \mu \mathrm{g}$ of oral misoprostol could prevent PPH in a community home-birth setting and to assess its acceptability and feasibility among rural Bangladeshi women.

Methods This quasi-experimental trial was conducted among women who had home delivery between November 2009 and February 2010 in two rural districts of Bangladesh. Two treatment arms included intervention group $(n=1009)$ receiving $400 \mu \mathrm{g}$ of misoprostol immediately after birth, and control group $(n=1008)$ without misoprostol. Primary PPH was measured by women's selfreported subjective measures of the normalcy of blood loss using the "cultural consensus model." Baseline data provided socioeconomic, reproductive, obstetric, and bleeding disorder information.

Findings The incidence of primary $\mathrm{PPH}$ was found to be lower in the intervention group (1.6\%) than the control group (6.2\%) $(\mathrm{p}<0.001)$. Misoprostol provided $83 \%$ protection (OR $0.17 ; 95 \%$ CI 0.05 to 0.54 ) from developing primary PPH. Women in the control group were more likely to need an emergency referral to a higher level facility and blood transfusion than the intervention group. Few women experienced transient side-effects of misoprostol. Eighty seven per cent of the women were willing to use misoprostol in their future pregnancy, and would recommend to other pregnant women.

Conclusion Community based distribution of oral misoprostol $(400 \mu \mathrm{g})$ appeared to be as effective, safe, acceptable, and feasible in preventing PPH in rural Bangladesh. This strategy may be scaled up across the country where access to skilled attendance is limited.

\section{6-5.6 SPONTANEOUS LIVE BIRTH AFTER IN VITRO FERTILISATION TREATMENT: FREQUENCY AND ASSOCIATED FACTORS}

doi:10.1136/jech.2011.142976b.89

\footnotetext{
1,2P Troude, ${ }^{* 1,3}$ E Bailly, ${ }^{4,5} \mathrm{~J}$ Guibert, ${ }^{2,3} \mathrm{~J}$ Bouyer, ${ }^{1,3} \mathrm{E}$ de La Rochebrochard. ${ }^{1} / \mathrm{Ined}$, Paris, France; ${ }^{2}$ Univ Paris Sud, Le Kremlin-Bicêtre, France; ${ }^{3}$ Inserm, CESP U1018, Le Kremlin-Bicêtre, France; ${ }^{4} A P-H P$, Hôpital Cochin, Paris, France; ${ }^{5}$ Institut Mutaliste Montsouris, Paris, France

Introduction Spontaneous live births (SLB) after in vitro fertilisation (IVF) treatment are not unusual, but reported SLB rates vary widely and little is known of their associated factors. Our objective was to examine the frequency of SLB and their associated factors among couples who had had IVF, successfully or unsuccessfully.
}

Methods A retrospective cohort of couples was recruited in eight French IVF centers. The couples had begun IVF treatment in the participating centers between 2000 and 2002, and were followed-up by postal questionnaire between 2008 and 2010. Analysis was carried out on 2134 couples who were still together at the time of the postal survey. Separate analyses were conducted according to the outcome of medical treatment: live birth $(n=1320)$ or no $(n=814)$. Multivariate analysis that included socio-demographic and medical characteristics was conducted using logistic regression.

Results The SLB rate was $17 \%$ among couples who had had a first live birth through medical treatment and $24 \%$ among couples who had unsuccessful treatment. In both groups, SLB was associated with younger age of the women, fewer IVF attempts and the origin of infertility. In couples who had not been successful with IVF, SLB was also associated with a shorter duration of infertility.

Conclusion Even in a population which had had IVF because of a very low monthly probability of conception, SLB is still possible, especially among couples who did not succeed in having a child through fertility treatment. Occurrence of SLB was mainly related to a better initial fertility prognosis.

\section{Tuesday 9 August 2011 IEA Regional Workshops JOINT WORKSHOP ORGANISED BY THE IEA REGIONS FOR NORTH AMERICA AND THE LATIN AMERICAN AND CARIBBEAN REGION}

\section{Chair: Dr Betty Monsour, USA \\ RW1-1 LINKING THE GLOBAL SOUTH AND NORTH IN THE AMERICAS: BUILDING HEMISPHERIC TIES AND SOLIDARITY TO PREPARE FOR THE 2014 IEA WORLD CONGRESS OF EPIDEMIOLOGY IN ALASKA: AMERICAN AND CARIBBEAN}

doi:10.1136/jech.2011.142976b.90

${ }^{1} \mathrm{~N}$ Krieger, ${ }^{*}{ }^{2} \mathrm{~B}$ Monsour, ${ }^{*} \mathrm{M}$ I Schmidt. ${ }^{*}$ Harvard School of Public Health, Boston, Massachusetts, USA; ${ }^{2}$ University of Alaska, Anchorage, Anchorage, Alaska, USA; ${ }^{3}$ School of Medicine, Federal University of Rio Grande do Sul, Porto Alegre, Porto Alegre, RS Brazil

The focus of this joint IEA Regional workshop, co-organised by the IEA North American Region and the IEA Latin American and Caribbean region, is "Linking the global South and North in the Americas: building hemispheric ties and solidarity to prepare for the 2014 IEA World Congress of Epidemiology in Alaska." Premised on the understanding that social justice is the foundation of public health, the purpose of our workshop is to bring together epidemiologists from across the Americas-as well as from any other region-to think together, imagine together, and work together to inspire ideas for presentations and perspectives of the upcoming IEA World Congress of Epidemiology to be held in Anchorage, Alaska in 2014. The working theme of WCE 2014 is Global Epidemiology in a Changing Environment: The Circumpolar Perspective. Creating a forum to address these issues, even from a circumpolar perspective, calls for an integrated approach from the Americas and the Caribbean, one that addresses the health status and needs of the region's myriad populations, including Indigenous peoples, immigrants, diverse racial / ethnic groups, and all those affected by social and economic deprivation and discrimination, in relation to not only race / ethnicity but also social class, gender, and sexuality. These are just a few of the issues we hope to investigate at WCE 2014. Please join us in this workshop opportunity as we plan for a truly global Congress. 